FORMULATION OF A FRAMEWORK FOR QUALITY ASSESSMENT OF PRECAST CONCRETE BASED ON 3D LASER SCANNING

*M. K. Kim, and H. Sohn

Korea Advanced Institute of Science and Technology

291Daehak-Ro Yuseong-Gu

Daejeon, South Korea 305701

(*Corresponding author: joekim@kaist.ac.kr)

D. Wu, J. C. P. Cheng, and C. C. Chang

The Hong Kong University of Science and Technology

Clear Water Bay

Kowloon, Hong Kong 


\title{
FORMULATION OF A FRAMEWORK FOR QUALITY ASSESSMENT OF PRECAST CONCRETE BASED ON 3D LASER SCANNING
}

\begin{abstract}
This study presents a framework for quality inspection of precast concrete components using the 3D laser scanning technique. As precast concrete-based rapid bridge construction is getting standardized, quality assessment of the precast concrete becomes critical for preventing any failures during the construction process. Moreover, as Building Information Modeling (BIM) gains popularity in the construction industry, autonomous and intelligent inspection systems are needed. Current method for quality control of precast concrete components, however, heavily relies on visual inspection and contacttype measurements, which are time and labor demanding. Also, storage and delivery systems of inspection information such as the procedures and results of quality inspection are lacking. To overcome these limitations of the current quality assessment technique for precast concrete, this study aims to develop a BIM-based framework for efficient quality inspection of precast concrete with the use of a 3D laser scanner. First, we formulate practical guidelines including detailed inspection procedure, selection of optimized scanner and scan location, inspection criteria, and data storage and delivery system. Second, to investigate the applicability of $3 \mathrm{D}$ laser scanning to precast concrete quality inspection based on the proposed framework, a case study inspecting quality of a lab-scale object assumed as precast concrete component is presented for detecting defects.
\end{abstract}

\section{KEYWORDS}

Precast concrete, Quality inspection, 3D laser scanning, Building information modeling

\section{INTRODUCTION}

Precast concrete has become more popular in the construction sector today. The main reasons of using precast concrete exist in cost, speed, and quality over conventional site-cast concrete method (Sacks et al., 2004). Moreover, because prefabrication is conducted in a well-controlled casting environment, the final products generally have high quality and uniformity. However, as precast concrete-based rapid construction is getting standardized, quality assessment of the precast concrete becomes critical for preventing any failures during the construction process. Current method for quality control of precast concrete components, however, heavily relies on visual inspection and contact-type measurements, which are time and labor demanding (Phares et al., 2004). In order to overcome these limitations, it is necessary to develop automated precast concrete inspection systems that can greatly reduce the manual work for inspectors and perform real-time quality assurance without human intervention.

There are a few studies aiming to adapt sensor systems to quality inspection of concrete structure. Among them, 3D laser scanning-based quality inspection has gained popularity in recent years. Anil et al. (2011) proposed a concrete structure quality inspection technique by conducting deviation analysis between as-design model and as-built model generated by a 3D laser scanner. Akinci et al. (2006) proposed a general framework of quality inspection on concrete structures including identifying inspection goal, inspection planning. Other quality inspection studies using 3D laser scanning have focused on detecting defects of concrete structures including large cracks, flatness, and volume of concrete defects (Teza et al., 2009; Tang et al., 2011; Olsen et al., 2011). However, these studies do not provide details on practical inspection frameworks for concrete structures such as selection of an optimal scanner, inspection procedure, and data delivery systems even though these factors are crucial to guarantee accurate and reliable inspection results. 
To tackle these shortcomings of current approaches, we aim to (1) develop a BIM-based framework for precast concrete quality inspection, and (2) conduct a case study to investigate the applicability of the proposed framework using a 3D laser scanner. For the former objective, four aspects consisting of (1) inspection procedure, (2) selection of an optimal scanner and scan location, (3) inspection goal, and (4) inspection data storage and delivery systems are included in the framework. For the case study, surface damages on a rectangular precast slab panel were detected based on the proposed framework.

\section{FRAMEWORK FOR PRECAST CONCRETE QUALTIY INSPECTION}

In this section, the proposed methodology framework for quality inspection of precast concrete are presented. The framework includes (1) detailed inspection procedure, (2) selection of scanner and scan location, (3) inspection goal, and (4) data storage and delivery.

\section{Inspection Procedure}

The main process of the quality inspection can be divided into 4 phases; (1) Supplying: The inspection samples are transported from the supplier to the inspection site. At the same time, information of material properties such as type and designed dimensions of precast concrete is stored in a BIM library. The inspection site can be the manufacturing company, pre-determined inspection location, or construction site. (2) Preparation: Before the inspection, samples are properly treated first. The precast component is conveyed and located at a pre-determined spot, and identification of the material information obtained from the BIM library is performed through portable electric devices such as smartphone and PDA. Then, scanner setup including scanner calibration and parameter setting is performed. (3) Scanning and Inspection: Once preparation for the scan is completed, acquisition of the scan data is followed. In this step, selection of ROI (Region of Interest) is conducted for effective data acquisition. After the scanning, feature extraction and filtering are implemented to automatically calculate the intended inspection criteria. Since the raw scan has a high data capacity, data reduction using data mining techniques is necessary for further processes. As a next step, the reference model which is designed by architects or engineers is compared with the as-built model created from the scan data. After the comparison, inspection results are obtained for each specific inspection criteria and stored in the BIM library. (4) Decision: The final decision whether the inspected component is accepted or not is classified based on comparison between the inspection results and the tolerance of the quality control criteria. If error of the results exceeds the tolerance, the precast sample is diagnosed as a failed product and is then disposed or returned. Otherwise, if the result is passed, the sample is delivered to construction sites.

\section{Selection of Scanner and Scan Location}

Selection of scanners is central to make quality inspection of precast concrete successful because the results of the quality inspection heavily depend on the scanner itself. Randall (2011) classified the applications of laser scanning for construction projects into four major categories in order of the level of detail, which are (1) rapid urban-scale mapping / modeling, (2) infrastructure asset management, (3) construction site monitoring, and (4) structural analysis and inspection. The standard for this classification is the accuracy requirements for each application. Among the four categories, this study, i.e. quality inspection, is involved where the accuracy is below the tolerance. Thus, selection of an optimal scanner for precast concrete quality inspection should be considered for meeting the requirements.

In this study, we consider five major factors for the selection of scanner (1) inspection tolerance, (2) measurement range, (3) accuracy, (4) price, and (5) scanning time, as shown in Figure 1. First, tolerance required for each inspection purpose should be clearly specified prior to selection of a scanner. The tolerance is specified in the (Precast Concrete Institute) manual (PCI, 2000). For instance, the tolerance for dimensions of general precast concrete slab is $\pm 6 \mathrm{~mm}$. Second, for selecting a scanner, measurement range of laser scanners should be investigated, since ideal scan distance is different for each commercially available scanner. The measurement range of a laser scanner is deeply related to working principles of laser scanners. There are mainly two types of working principle, i.e. TOF (Time of Flight) 
and phase-shift principles. Typically, TOF scanners have relatively long measurement range (up to $1000 \mathrm{~m}$ ) compared to those of phase shift scanners (up to $120 \mathrm{~m}$ ) (RIEGL, 2013; FARO, 2013). Accuracy of a scanner is another important factor for selection of a proper scanner. In a similar manner of measurement range, accuracy is dependent on the working principle of the laser scanner. Phase shift scanners generally give better accuracy of around $2-3 \mathrm{~mm}$ than those of TOF scanner. Another standard for selecting a scanner is price, which is more realistic matter for inspectors. Commercially available laser scanners are quite expensive compared to other sensors such as vision camera or GPR (Ground Penetrating Radar) sensors. The price ranges from US\$30,000 to US\$200,000. The last factor for scanner selection is scanning time. For speedy and instantaneous quality decision, less scanning time is preferred. The scanning duration depends on horizontal and vertical speeds of the scanner. Also, size of scan objects affects the scanning time. The bigger the dimension of a precast concrete panel is, the more scanning time is required. Moreover, scanning time is affected by extrinsic and intrinsic scan parameters such as scan distance, angular resolution and incident angle.

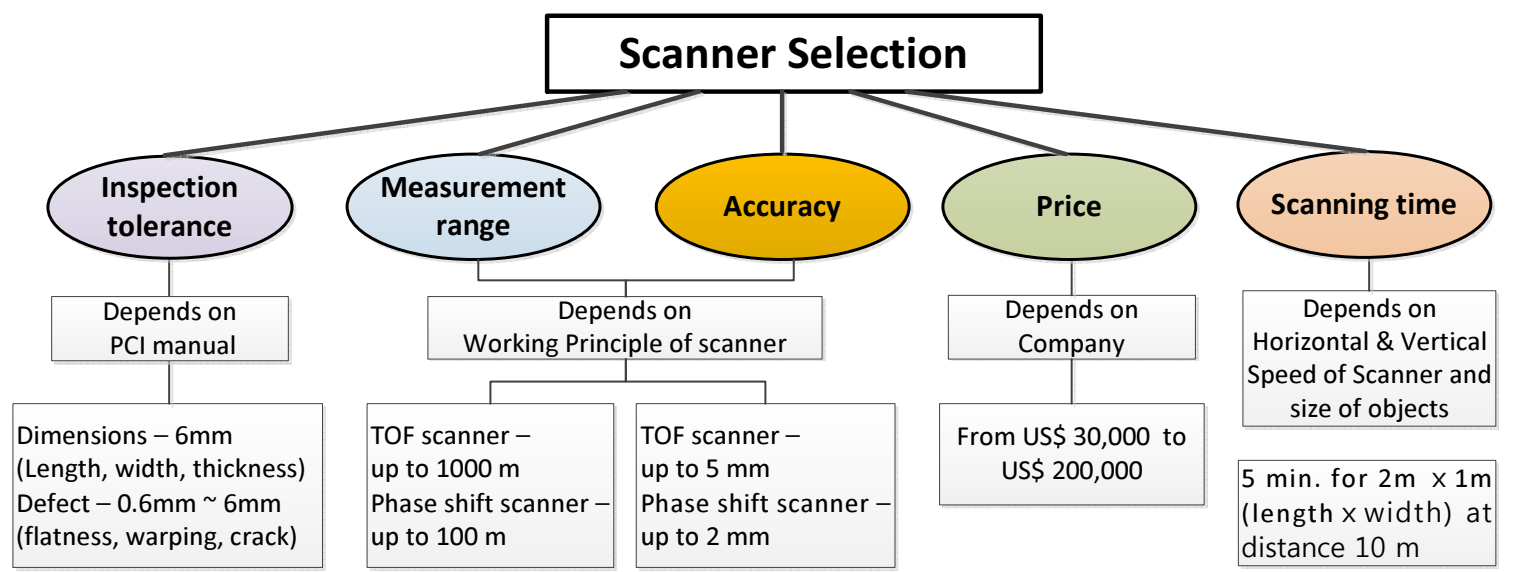

Figure 1 - Criteria of scanner selection for precast concrete quality inspection

Considering all these five criteria, an optimization equation minimizing the total inspection cost can be formulated by adding each factor having different weighting factors. The weighting factors for each criterion are differently determined with respect to inspection purposes. For example, for the dimensional quality inspection requiring tolerance of $\pm 6 \mathrm{~mm}$, accuracy is the most important factor for scanner selection so that the weighting factor for accuracy should be higher than those of the other criteria.

Moreover, selection of an optimal scanning position is important to obtain the most accurate and reliable inspection quality while minimizing cost and time. In order to minimize cost and time, the fewest number of scans is preferred for acquisition of an object's geometric information. Also, dense scan points are easier to obtain more accurate quality results than sparse scan points. However, since dense scan points require much scanning time and computing cost, a model for optimizing scanner location needs to be formulated to meet both demands.

\section{Inspection Goal}

Before acquisition of scan data, determination of inspection goals should be preceded. There are two categories in the inspection goal, i.e. geometric and defect characteristics. These inspection items are selected from the checklists and inspection manual of PCI (PCI, 2000). Table 1 shows the inspection goals. First, geometric properties consisting of dimension, straightness and squareness are inspected. For the detailed inspection goals, dimensional quality such as length amd width of precast concrete panels is selected. As for the second inspection goal, defect properties include pit, crack, warping, and flatness. Subinspection goals for each attribute are also selected for the inspection criteria. For example, for pit inspection, three items, i.e. number, volume, area and location, are the detailed inspection goals. 
Table 1 - Inspection goals for precast concrete

\begin{tabular}{lll}
\hline Quality Criteria & Attributes & Description \\
\hline \multirow{2}{*}{ Geometric } & Dimension & Length; Width; Thickness \\
Characteristics & Straightness & Size; Location \\
& Squareness & Amount; Location \\
\hline \multirow{3}{*}{ Defect } & Pit & Volume; Area; Location \\
Characteristics & Crack & Depth; Length; Width; Location \\
& Warping & Volume; Size; Location \\
& Flatness & Size (Depth); Location \\
\hline
\end{tabular}

\section{Data Storage and Delivery}

In most projects in the civil engineering sector, the construction data are stored and delivered within various formats due to the diversity of participants. For example, as applied to this study, scan data consisting $\mathrm{x}, \mathrm{y}$, and $\mathrm{z}$ coordinate information are stored in ASCII, whereas AutoCAD designers use DWG files to draw the scanned projects. This data storage and delivery unavoidably generates inconsistency of data or even information loss. For the inspection process of precast concrete, the same inspection result may sometimes have different quality levels, due to the information asymmetry. Thus, it is necessary to transform inspection data into a standardized form, like the Industry Foundation Classes (IFC) format. The IFC data is an object-oriented data model based on class definitions representing the details (elements, shapes, processes, etc.) and it is widely utilized in applications of construction or facility management project. Figure 2 describes the concept of the proposed data storage and delivery system. All information created during all processes of the inspection is stored in a BIM library through cloud sever. The information is represented as an IFC file so that all participants having access authority can update and access those information without limitation of location and time.

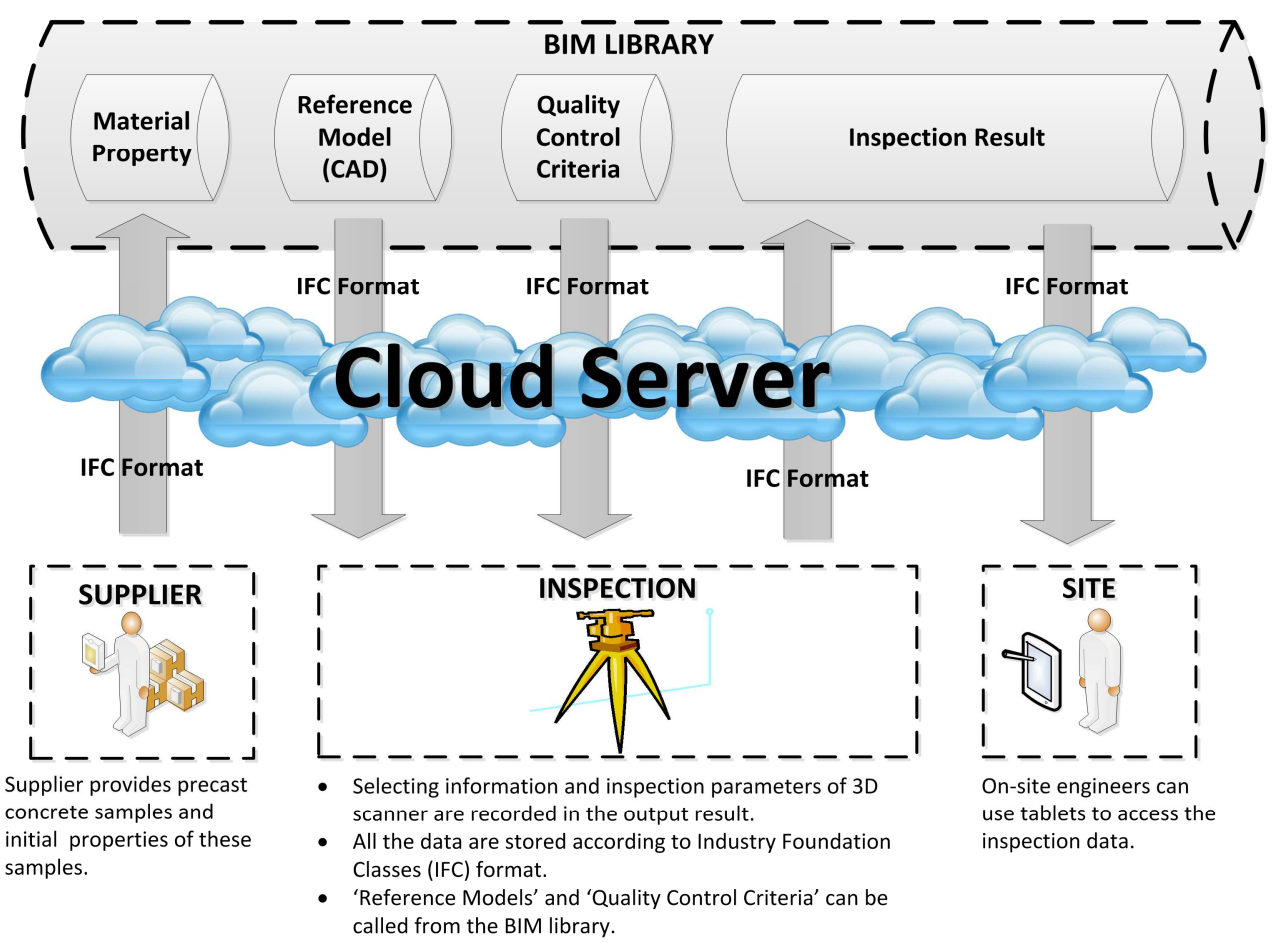

Figure 2 - Scheme of the data storage and delivery system

To represent and store the inspection data in the IFC format, an IFC extension is proposed. Using an Entity-Relationship Model (ER Model), Figure 3 illustrates the association between different entities in the proposed IFC extension. For example, the entity 'IfcPit' which is the sub-type of 'IfcInspectionResult', 
has three attributes, i.e. number, area, volume, and location. The schema of the 'IfcPit' entity is shown in Figure 3. Note that due to the limited space, not all the element attributes in the entity are included in Figure 3.

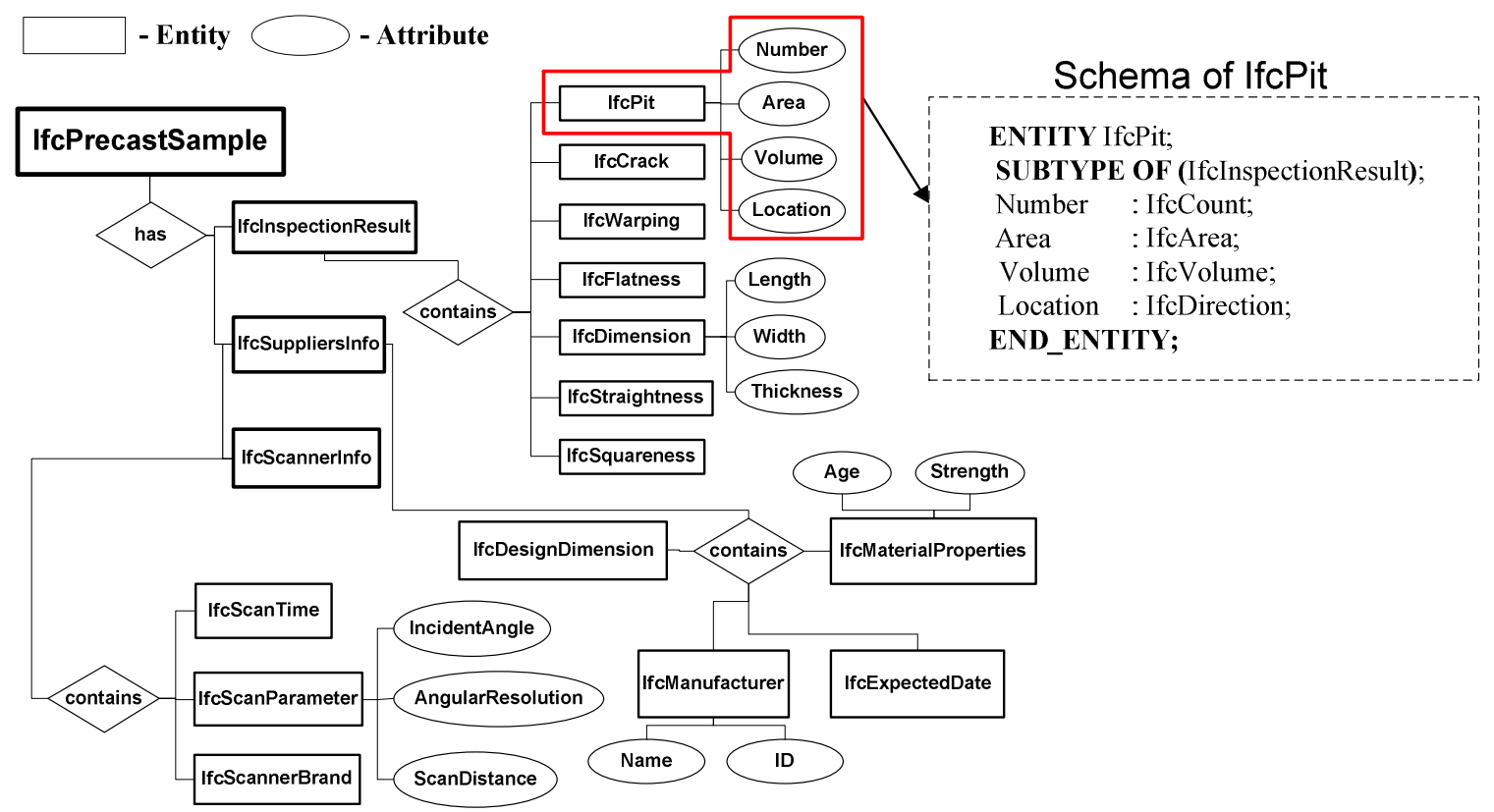

Figure 3 - IFC entity illustration for a precast concrete sample using ER model

\section{CASE STUDY}

The objective of this case study is to recognize precast concrete surface defects quantitatively using a 3D laser scanner and identify the applicability of the proposed framework. For data analysis using the obtained scan data, a damage localization technique utilizing damage sensitive features was developed. Also, damage volume estimation was performed to quantify the recognized damages.

\section{Experimental Configuration}

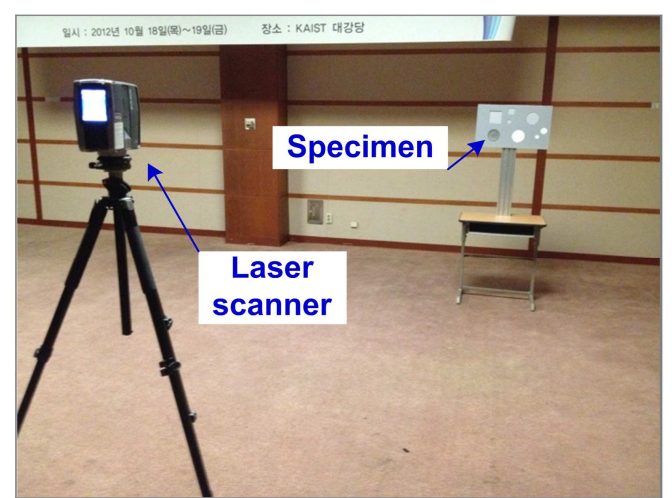

(a) Test configuration

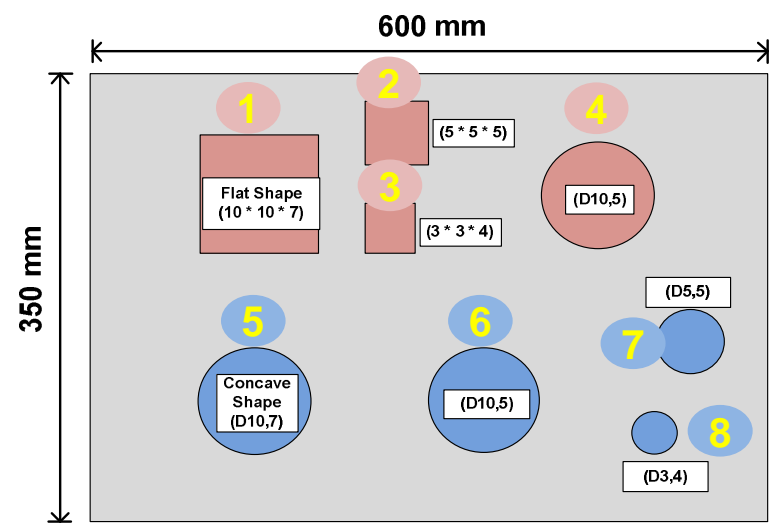

(b) Test specimen

Figure 4 - Experimental set-up and test specimen

Figure 4 shows the test configuration and the test specimen. The scanner, FARO Focus-3D, was selected based on the framework for the scanner selection. Since this study aims to detect concrete surface 
defects with level of below $4 \mathrm{~mm}$, the first priority was the accuracy of the scanner. The scanner provides an accuracy of $3 \mathrm{~mm}$ at a distance $10 \mathrm{~m}$, which are the best among commercial scanners (FARO Inc. 2013). The laser scanner was mounted on a tripod and scanned the test specimen both horizontally and vertically as shown in Figure 4(a). Two different parameters for both distances $(8 \mathrm{~m}$ and $12 \mathrm{~m})$ and angular resolutions $\left(0.009^{\circ}\right.$ and $\left.0.018^{\circ}\right)$ were investigated in this study. The test specimen made of Styroform was assumed as a precast concrete panel. The eight surface defects with varying size and thickness were induced as shown in Figure 4(b). For the damages, two types of damage shape, i.e. flat (numbered from 1 to 4 ) and convex (numbered from 5 to 8 ) pit damages, were simulated to mimic practical surface damages of the precast concrete.

\section{Data Analysis}

\section{Procedure}

It started with acquisition of spatial information of the object from the laser scanner. Then, coordinate transformation from scanner coordinate to object coordinate was performed. This process made one of the corner points of the object locate at the origin $(0,0,0)$ and it helped computation cost for the posterior processes. Next, filtering of unwanted background data was performed by setting bounds to the coordinate transformed data. Sequentially, damage localization was implemented by using two damage sensitive features. Finally, damage quantification on localization and volume estimation of the damage was performed.

\section{Damage Sensitive Features}

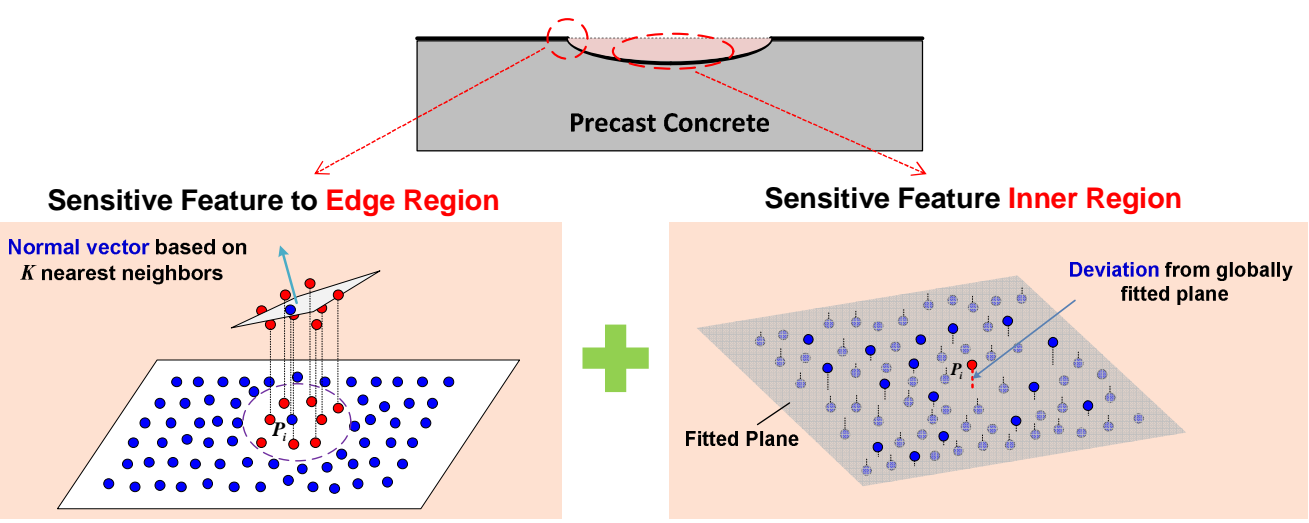

(a) Normal vector variation of locally fitted plane

(b) Deviation from globally fitted plane

Figure 5 - Two damage sensitive features

For damage localization, two features, (1) normal vector variation of locally fitted plane and (2) deviation from globally fitted plane were used in this study as shown in Figure 5. First, normal vector variation of locally fitted plane was employed for detection of edge region of damages. To obtain normal vector of a scan point, $k$-nearest neighbors of the scan point were firstly extracted based on Euclidean distance. Then, a local surface was created by fitting those extracted neighbors such that summation of the deviations between the fitted plane and each point was minimized. In this study, $k$ was determined as eight since it represented the average normal vector of the local points situated at all directions. Generally, in edge region of damage area, variation of normal vector of locally fitted plane is larger than those of undamaged area. From this observation, a damage index based on variation of normal vector of locally fitted plane can be formulated. Second, deviation from the globally fitted plane was used to detect the inner region of damage. In Figure 5(b), deviation of a scan point can be defined as the closest distance between the point and the fitted plane. In general, deviation in the inner region has larger values than those of other intact regions. From this observation, damage index based on deviation from the globally fitted plane can be formulated. To detect the damage area accurately, these two damage features are combined together 
such that the combined damage index is both sensitive to the edge and inner damage area. Thus, the combined damage index can be determined as:

$$
D I_{\text {Comb }}\left(p_{i}\right)=\max \left(D I_{\text {Var_normal }}\left(p_{i}\right), D I_{\text {Dev }}\left(p_{i}\right)\right)
$$

where $D I_{C o m b}\left(p_{i}\right)$ denotes combined damage index, and $D I_{\text {Var_normal }}\left(p_{i}\right)$ and $D I_{\text {Dev }}\left(p_{i}\right)$ stand for normal vector variation and deviation damage indices, respectively. By taking maximum value between two damage indices, robust damage localization can be achieved. In a similar manner, threshold value, $T R$, is determined by taking maximum between thresholds of two damage indices as:

$$
T R=\max \left(T R_{V a r \_n o r m a l}, T R_{\text {Dev }}\right)
$$

The threshold of two damage indices, $T R_{\text {Var_normal }}$ and $T R_{D e v}$, can be obtained from probability density function of Weibull distribution of calculated damage indices in undamaged area. For real applications, threshold can be determined before initiation of scanning by conducting a pre-scanning on the healthy surface of a precast concrete panel. Therefore, a damage classifier can be determined as:

$$
\text { If } \operatorname{DI}_{\text {Comb }}\left(p_{i}\right)>T R \text {, then } p_{i} \text { is diagnosed as damaged point }
$$

\section{Experimental Result}

\section{Damage Localization and Quantification}

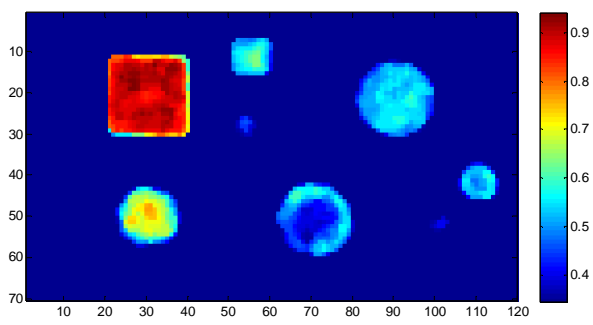

(a) Image of combination damage index

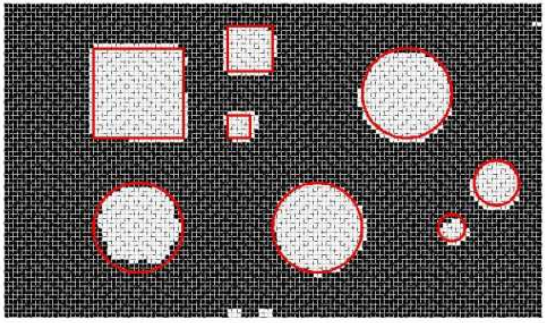

(b) Image of damage classification

Figure 6 - Damage localization results of the experiment

Table 2 - Damage quantification on localization

\begin{tabular}{cccccccc}
\hline \multirow{2}{*}{ Distance $(\mathrm{m})$} & Angular & \multicolumn{2}{c}{ Damage 1 } & \multicolumn{2}{c}{ Damage 5 } & \multicolumn{2}{c}{ Damage 8 } \\
& resolution $\left(^{\circ}\right)$ & Recall & Precision & Recall & Precision & Recall & Precision \\
\hline \multirow{2}{*}{8} & 0.009 & 1.00 & 0.86 & 0.78 & 0.99 & 0.62 & 0.62 \\
& 0.018 & 1.00 & 0.80 & 0.90 & 0.95 & 0.93 & 0.60 \\
\multirow{2}{*}{12} & 0.009 & 1.00 & 0.84 & 0.94 & 0.95 & 0.68 & 0.60 \\
& 0.018 & 1.00 & 0.71 & 0.99 & 0.86 & 0.93 & 0.57 \\
\hline
\end{tabular}

Table 3 - Damage quantification on volume estimation

\begin{tabular}{ccccc}
\hline Distance $(\mathrm{m})$ & Angular resolution $\left(^{\circ}\right)$ & Damage 1 & Damage 5 & Damage 8 \\
\hline \multirow{2}{*}{8} & 0.009 & 0.99 & 0.79 & 0.74 \\
& 0.018 & 0.97 & 0.84 & 0.95 \\
\multirow{2}{*}{12} & 0.009 & 0.98 & 0.94 & 0.70 \\
& 0.018 & 0.95 & 0.90 & 0.75 \\
\hline
\end{tabular}

Figure 6 shows the damage localization results. With combination of two damage features, complementary result was obtained in Figure 6(a). For damage diagnosis, a threshold value was determined by Weibull distribution of the damage indices in undamaged area with confidence interval of $97 \%$. Figure 6(b) shows the damage classification results with the actual damage area (red line). Tables 2 and 3 show the damage quantification results on localization and volume estimation, respectively. For damage localization, recall and precision rates which are commonly used in quantifying classification performance were employed. The recall rate stands for the ratio of correctly detected damage area over actual damage area, whereas the 
precision rate stands for the ratio of correctly detected damage area over detected damage area. Note that due to space limitation, only three damage cases, damages 1, 5 and 8, were presented in both Tables 2 and 3 . The result shows that recall and precision rates of flat-type damage (damage 1) are relatively higher than those of concave-type damage (damages 5 and 8). Also, it is shown that both location and volume estimation results for damage 8 with defect of $3 \mathrm{~cm}$ diameter and $4 \mathrm{~mm}$ thickness are low compared to the other cases. It indicates that small amount of damage, which is comparable to the accuracy level of the laser scanner, has difficulty to be detected.

\section{$\underline{\text { Data Storage and Delivery of Inspection Result }}$}

The inspection results obtained from the previous damage location and quantification stages can be stored in the IFC representation as mentioned in the section of framework formulation. For instance, the inspection result for pit damage of a precast concrete sample was stored and delivered as 'IfcPit', which has four attributes (number, area, volume, and location of the pit damage) in our proposed IFC extension. The following schema tree shows the inspection data for pit damage obtained from the previous experiment.

$\# 288=$ IfcPit $(\# 276, \# 277, \# 10, \# 6)$

\#276=IfcCount $(8)$;

\#277=IFCArea $((115.5,30.5,4.8,83.8,61.0,81.0,24.0,7.3)$, 'cm2');

$\# 10=$ IFCVolume $\left((74.9,11.8,3.1,31.3,27.1,27.8,7.2,1.4),{ }^{\prime} \mathrm{cm} 3\right.$ ');

\#6=IFCDirection $(((15.0,10.0),(27.5,5.0),(26.3,13.8),(42.5,10.0),(15.0,25.0),(35.0,25.0),(55.0,20.0)$, $(50.0,25.0)))$;

\section{CONCLUSIONS}

This paper describes a BIM-based framework for efficient precast concrete quality inspection using 3D laser scanning. Four aspects consisting of (1) inspection procedure, (2) selection of scanner and scan location, (3) inspection goal, and (4) data storage and delivery system are investigated for the framework. To verify the proposed framework, a case study using a 3D laser scanner was conducted and quantification of damages was successfully estimated. The case study demonstrated that this study can contribute to advances in autonomous and rapid precast concrete quality inspections.

\section{REFERENCES}

Akinci, B., F. Boukamp, C. Gordon, D. Huber, C. Lyons \& K. Park (2006). A formalism for utilization of sensor systems and integrated project models for active construction quality control. Automation in Construction, 15(2), 124-138.

Anil, E. B., P. Tang, B. Akinci \& D. Huber (2011). Assessment of quality of as-is building information models generated from point clouds using deviation analysis. Proceedings of SPIE, ThreeDimensional Imaging, Interaction, and Measurement(78640F), San Francisco, California, USA

FARO (2013), Focus-3D Technical Specification, FARO Inc.

Olsen, N.J., Kuester, F., Chang, B.J. \& Hutchinson, T.C. (2010). Terrestrial laser scanning-based structural damage assessment. Journal of Computing in Civil Engineering, 24 (3), 264-272.

PCI (2000), Tolerance Manual for Precast and Prestressed Concrete Construction, Precast/Prestressed Concrete Institute, (Reported by PCI committee)

Phares, B., Washer, G., Rolander, D., Graybeal, B. \& Moore, M. (2004). Routine highway bridge inspection condition documentation accuracy and reliability. Journal of Bridge Engineering, 9 (4), 403-413

Randall T (2011). Construction engineering requirements for integrating laser scanning technology and building information modeling. Journal of Construction Engineering and Management, 137(10), 797-805.

RIEGL (2013), VZ-400 Technical Specification, RIEGL Inc.

Sacks, R., Eastman, C. M., \& Lee, G. (2004). Process model perspectives on management and engineering procedures in the precast/prestressed concrete industry. Journal of Construction Engineering Management, $130(2), 206-215$.

Tang, P., Hubber, D. \& Akinci B. (2011). Characterization of laser scanners and algorithms for detection flatness defects on concrete surfaces. Journal of Computing in Civil Engineering, 25 (1), 31-42.

Teza, G., Galgaro, A. \& Moro, F. (2009). Contactless recognition of concrete surface damage from laser scanning and curvature computation. NDT\&E International, 42 (4), 240-249. 